\title{
Epigenetic inactivation of ADAMTS9 via promoter methylation in multiple myeloma
}

\author{
LING PENG ${ }^{1,2}$, ZESONG YANG $^{1,3}$, CUI TAN $^{2}$, GUOSHENG REN $^{2}$ and JIANBIN CHEN ${ }^{1}$ \\ ${ }^{1}$ Department of Hematology; Laboratories of ${ }^{2}$ Molecular Oncology and Epigenetics and ${ }^{3}$ Hematology, \\ The First Affiliated Hospital of Chongqing Medical University, Chongqing 400016, P.R. China
}

Received November 10, 2012; Accepted January 21, 2013

DOI: $10.3892 / \mathrm{mmr} .2013 .1291$

\begin{abstract}
A disintegrin-like and metalloprotease with thrombospondin type I motifs (ADAMTS) are a family of 19 secreted mammalian metalloproteases. ADAMTS9 was reported to be a novel tumor suppressor gene and is downregulated in various types of human cancer due to hypermethylation of promoter CpG islands. In the present study, the silencing mechanism of the ADAMTS9 gene was analyzed in the multiple myeloma (MM) cell lines, KM3 and RPMI-8226. Control and MM samples were obtained by conventional bone marrow (BM) biopsy of normal and MM adult BM, respectively. RT-PCR revealed a high expression of the ADAMTS9 gene in normal samples and RPMI-8226 cells while marked gene silencing of ADAMTS9 was observed in MM patients and KM3 cells. Promoter methylation of ADAMTS9 was detected in the KM3 cell line and 66\% (37/56) MM patients by methylation-specific PCR. In addition, the DNA demethylating agent, 5-aza-2'-deoxycytidine and trichostatin A restored ADAMTS9 expression by suppressing promoter methylation in KM3 cells. Ectopic expression of ADAMTS9 in ADAMTS9-silenced MM cells was found to significantly suppress cell colony formation and proliferation. In the present study, DNA methylation was found to play a key role in ADAMTS9 gene silencing and the biological behavior of myeloma cells. The results demonstrate that ADAMTS9 silencing by methylation may be a novel tumor marker for MM and the applicability of demethylating agents in the treatment of MM.
\end{abstract}

\section{Introduction}

Multiple myeloma (MM) is a plasma cell malignancy and the second most common hematological malignancy after non-Hodgkin lymphoma (1). Despite advances in supportive and systemic therapies, MM remains an essentially incurable

Correspondence to: Dr Jianbin Chen, Department of Hematology, The First Affiliated Hospital of Chongqing Medical University, 1 Yuanjiagang Friendship Road, Chongqing 400016, P.R. China

E-mail: cqchenjianbin2007@126.com

Key words: multiple myeloma, epigenetic, promoter methylation, ADAMTS9, KM3 disease with a high rate of relapse and a rapid acquisition of drug resistance. The development of $\mathrm{MM}$ is a multistep process associated with an increasing frequency of genetic aberrations, including chromosomal abnormalities, as well as complex translocations, including the complete deletion of chromosome 13 or its long arm. In addition, increased density of bone marrow (BM) microvessels is associated with MM development (2-4).

However, an increasing number of studies are reporting that, in addition to genetic abnormalities, epigenetic processes play a major role in the carcinogenesis of MM $(5,6)$. DNA methylation of cytosine bases within a $\mathrm{CpG}$ dinucleotide represents a major epigenetic modification that results in altered transcriptional activity in the molecular pathogenesis of various hematological malignancies (7). In MM, numerous cases of epigenetic silencing of tumor suppressor genes (TSGs), including p15, p16, DAP-kinase, CDH1, DLC1, SOCS-1 and E-Cadherin, have been reported (8-11). In addition, a member of the a disintegrin-like and metalloprotease with thrombospondin type I motifs (ADAMTS) family has been suggestedc to be involved in processes regulating epigenetic gene silencing in MM.

The ADAMTS superfamily is involved in a wide range of cellular processes, including the maturation of procollagen and the extracellular matrix proteolysis associated with morphogenesis, cancer, angiogenesis, arthritis and ovulation. Several members of the ADAMTS family (ADAMTS9, ADAMTS12, ADAMTS15 and ADAMTS18) have been reported as candidate TSGs.

ADAMTS9, which is located at 3p14.2, is the most highly conserved member of this superfamily. ADAMTS9 is involved in a number of biological processes, including melanoblast development, vascular development and the suppression of angiogenesis in the tumor context (12). The gene functions as a TSG via promoter methylation in various types of human cancer, such as esophageal, nasopharyngeal, gastric, colorectal and pancreatic (13-15).

In the present study, the expression and promoter methylation status of ADAMTS9 was analyzed in MM cell lines and patients. Promoter hypermethylation and the re-expression of ADAMTS9 were performed using demethylating drug treatment and results revealed that promoter methylation is the key mechanism of ADAMTS9 expression inactivation in MM. Ectopic ADAMTS9 expression in KM3 cells leads to the 
significant suppression of colony formation and cell proliferation, indicating that ADAMTS9 is a novel functional tumor suppressor gene.

\section{Materials and methods}

Cell culture and clinical samples. KM3 and RPMI-8226 cell lines were obtained from Dr Jian Hou (The Second Military Medical University, Shanghai, China) and maintained in RPMI-1640 medium supplemented with 10\% fetal bovine serum (both Gibco-BRL, Carlsbad, CA, USA). A total of 32 male and 24 female patients with a median age of 64 (range, 38-78) years, recently diagnosed with MM, as well as 16 healthy adults, who served as controls, were included in the study. Patients were previously untreated. BM samples were obtained from the Hematology Laboratory of The First Affiliated Hospital of Chongqing Medical University (Chongqing, China). The diagnosis of MM was based on standard criteria (International Myeloma Working Group, 2003) and the patients were classified according to the International Staging System (ISS). The samples were confirmed to have no solid tumor infiltration. The study was conducted according to the principles of the Declaration of Helsinki and informed consent was obtained from all patients.

Methyltransferase inhibitor drug treatment. Cells were treated with $10 \mu \mathrm{M}$ 5-aza-2'-deoxycytidine (Aza; Sigma-Aldrich, St. Louis, MO, USA) for 3 days and then with $100 \mathrm{ng} / \mathrm{ml}$ trichostatin A (TSA; Sigma-Aldrich) for an additional $24 \mathrm{~h}$, as described previously (16).

Total RNA isolation and semi-quantitative reverse transcription PCR (RT-PCR). RNA was extracted using TRIzol reagent (Invitrogen Life Technologies, Carlsbad, CA, USA) and reverse-transcribed using an RT reagent kit (Takara Bio, Inc., Shiga, Japan) and random hexamer primers. PCR analysis using Go-Tag (Promega Corporation, Madison, WI, USA) was perfomed using GAPDH as a control. ADAMTS9 expression was analyzed by PCR using the following primers: ADAMTS9, F: 5'-CAT GCA GTT TGT ATC CTG-3' and R: 5'-GCG TTC TTT TGA AGT GGA CG -3'; GAPDH, F: 5'-ATC TCT GCC CCC TCT GCT GA-3' and R: 5'-GAT GAC CTT GCC CAC AGC CT-3'. RT-PCR was performed with 32 cycles for ADAMTS9 and 23 cycles for GAPDH.

DNA bisulfite treatment and methylation-specific polymerase chain reaction (MSP). Genomic DNA was extracted using the QIAmp DNA blood Mini kit (Qiagen, Hilden, Germany) according to the manufacturer's instructions. Bisulfite modification of DNA and methylation of the $\mathrm{CpG}$ islands of the ADAMTS9 promoter were performed as described previously (13). The primers detecting the methylated or unmethylated alleles of the ADAMTS9 promoter were as follows: ADAMTS9-m1: 5'-TTT TTC GTT TTT TTT TGT TCG TTC-3' and -m2: 5'-AAA CTA AAC CGC TCG AAC CG-3' for the methylated alleles and ADAMTS9-u1: 5'-GTT TTT TGT TTT TTT TTG TTT GTT T-3' and -u2: 5'-AAA AAC TAA ACC ACT CAA ACC A-3' for the unmethylated alleles. MSP was performed for 40 cycles using Ampli Taq-Gold under methylation- and unmethylation-specific primer conditions: annealing temperature 60 and $58^{\circ} \mathrm{C}$, respectively. The MSP primers were tested previously to ensure that DNA that was not modified by bisulfite was not amplified.

Cell transfection. Transfection plasmids were purified using the TIANprep Mini Plasmid kit [Tiangen Biotech (Beijing) Co., Ltd., Beijing, China]. KM3 cells $\left(2 \times 10^{5}\right.$ cells/well) were plated in 6-well plates and transfected with the expression plasmid $(2 \mu \mathrm{g}), \mathrm{pCEP} 4-A D A M T S 9$ or the empty vector $(2 \mu \mathrm{g})$, pCEP4, using Lipofectamine 2000 (Invitrogen Life Technologies) according to the manufacturer's instructions. Cells were collected and plated in a $5-\mathrm{cm}$ dish $48 \mathrm{~h}$ post-transfection and selected for 21 days with G418 $(0.4 \mathrm{mg} / \mathrm{ml})$.

Colony formation assay. Colony formation assay was performed using semi-solid medium. Cells were suspended in RPMI-1640 medium containing 1\% methylcellulose, $35 \%$ fetal bovine serum and $0.8 \mathrm{mg} / \mathrm{ml} \mathrm{G} 418$ in a $5-\mathrm{cm}$ dish $48 \mathrm{~h}$ post-transfection. The dish was placed in a sealed chamber and incubated at $37^{\circ} \mathrm{C}$ in a $5 \% \mathrm{CO}_{2}$ incubator for 21 days. The number of surviving colonies ( $\geq 50$ cells $/$ colony) $/ \mathrm{cm}^{3}$ was quantified using an inverted microscope. Total RNA from the transfected cells was extracted and analyzed via RT-PCR to confirm the ectopic expression of ADAMTS9. The experiments were performed in triplicate wells 3 times.

Flow cytometric analysis of the cell cycle. KM3 cells were plated in 6-well plates and transfected with $4 \mu \mathrm{g}$ pCEP4-ADAMTS9 or the empty vector, pCEP4, using Lipofectamine 2000 according to the manufacturer's instructions. Stable ADAMTS9 expression and vector-KM3 cells were harvested and fixed in ice-cold 70\% ethanol for $1 \mathrm{~h}$. Cell cycle profiles were assayed using an Elite ESP flow cytometer and the data were analyzed using CellQuest software (BD Biosciences, Franklin Lakes, NJ, USA).

Measurement of cell proliferation. The impact of the ectopic expression of ADAMTS9 on KM3 cell proliferation was assessed using the Cell Counting kit-8 (CCK-8) (Beyotime Institute of Biotechnology, Jiangsu, China) according to the manufacturer's instructions. Briefly, KM3 cells were transfected as described above and cultured at a density of $3 \times 10^{3}$ cells/ well in 96-well plates. Cell proliferation was monitored at 24 , 48 and $72 \mathrm{~h}, 20 \mu \mathrm{l}$ CCK- 8 solution was added to each well following $1 \mathrm{~h}$ incubation in a $5 \% \mathrm{CO}_{2}$ humidified incubator at $37^{\circ} \mathrm{C}$ and the optical density was measured at $450 \mathrm{~nm}$ using a microplate reader (Molecular Devices LLC, Sunnyvale, CA, USA). Experiments were performed in 5 wells/experiment and repeated 3 times.

Statistical analysis. Statistical analyses were performed using SAS version 9.1 for windows (SAS Institute Inc., Cary, NC, USA). Data were presented as the mean \pm SD. Associations between the ADAMTS9 methylation status of the tumor and non-tumor samples and the clinical characteristics were assessed using Chi-square, Fisher's exact and Student's t-tests. Differences between the subgroups (colony formation assay, cell cycle analysis and proliferation measurement) were assessed using the Student's test. $\mathrm{P}<0.05$ was considered to indicate a statistically significant difference. 
Table I. Methylation status of ADAMTS9 promoter in MM ( $\mathrm{n}=56)$.

ADAMTS9 promoter

Samples

Methylated

Unmethylated

Frequency of methylation (\%)

Multiple myeloma, $\mathrm{n}$

37

19

$37 / 56(66)$

ADAMTS9, a disintegrin-like and metalloprotease with thrombospondin type I motifs 9; MM, multiple myeloma.
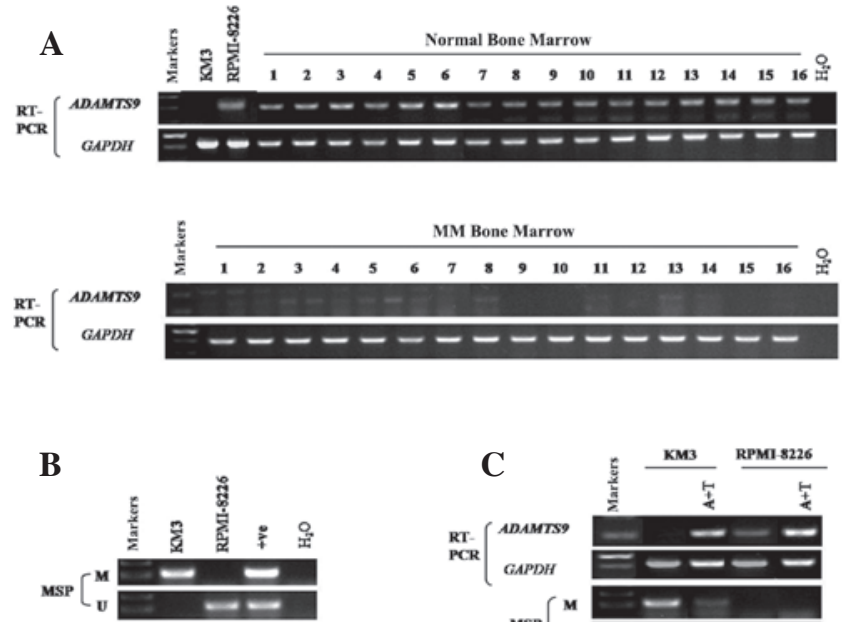

Figure 1. Downregulation of ADAMTS9 in KM3 MM cells. (A) Semi-quantitative RT-PCR of expression levels of ADAMTS9 in RPMI-8226 cells and human normal samples. ADAMTS9 was downregulated in KM3 MM cells and MM samples while high expression levels of ADAMTS9 were observed in RPMI-8226 cells and human normal samples. GAPDH was used as a control. (B) MSP of ADAMTS9 methylation status in KM3 and RPMI-8226 cells. (C) RT-PCR of ADAMT59 expression in KM3 cells following treatment with A+T. MSP of ADAMTS9 methylation status in KM3 and RPMI-8226 cells prior to and following A+T treatment. KYSE510M was used as a positive control. ADAMTS9, a disintegrin-like and metalloprotease with thrombospondin type I motifs 9; MM, multiple myeloma; MSP, methylation-specific PCR; M, methylated ; U, unmethylated; A+T, 5-aza-2'-deoxycytidine and trichostatin A.
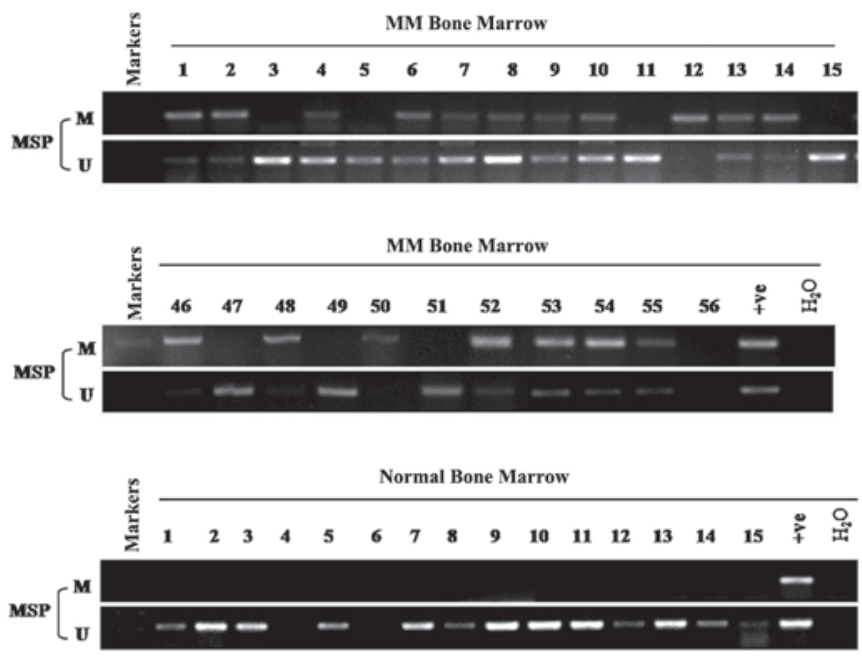

Figure 2. ADAMTS9 CpG methylation in MM bone marrow and normal samples. KYSE510M was used as a positive control. ADAMTS9, a disintegrin-like and metalloprotease with thrombospondin type I motifs 9; MM, multiple myeloma; M, methylated ; U, unmethylated.

\section{Results}

ADAMTS9 was expressed in healthy normal adult tissues and frequently silenced in MM cell lines and patient samples. Expression levels of ADAMTS9 were analyzed in KM3 and RPMI-8226 cells, 16 MM BM samples and 16 normal adult BM samples with semi-quantitative RT-PCR. The results revealed that ADAMTS9 was silenced in $100 \%(16 / 16)$ of the MM samples (Fig. 1A), while its expression was readily detected in the normal adult BM samples (0/16) (Fig. 1A). In addition, among the two MM cell lines tested, KM3 revealed no ADAMTS9 expression (Fig. 1A). By contrast, ADAMTS9 expression was detected in RPMI-8226 cells (Fig. 1A).

Frequent silencing of ADAMTS9 in MM cell lines due to promoter methylation. As aberrant promoter $\mathrm{CpG}$ methylation is associated with gene silencing, the methylation status of the MM cell lines was determined using MSP. ADAMTS9 was methylated in KM3 (Fig. 1B), while methylation was not observed in RPMI-8226 cells and normal adult BM (Figs. 1B and 2), consistent with the hypothesis that ADAMTS9 methylation is negatively correlated with ADAMTS9 expression levels.

Activation of ADAMTS9 expression via pharmacological demethylation. To examine whether promoter methylation of ADAMTS9 directly mediates expression silencing, cells were treated with the DNA methyltransferase inhibitor, Aza, combined with the histone deacetylase inhibitor, TSA. ADAMTS9 expression was markedly induced following drug treatment in KM3 cells (Fig. 1C), accompanied with a decrease in methylated alleles and an increase in unmethylated alleles of the ADAMTS9 promoter in KM3 cells (Fig. 1C). However, no difference was observed in RPMI-8226 cells (Fig. 1C). The results indicate that DNA methylation of the ADAMTS9 promoter is directly involved in transcriptional silencing in MM cells.

Frequent ADAMTS9 methylation and clinicopathological correlations in MM. MSP was used to analyze the methylation status of ADAMTS9 in $56 \mathrm{MM}$ and 15 normal adult BM samples. Aberrant promoter methylation was detected in 66\% (37/56) of the MM BM samples (Table I, Fig. 2), while no methylation was detected in any of the 15 normal adult samples (Fig. 2). Potential correlations between the ADAMTS9 methylation status and clinical parameters were determined, including gender, age, MM subtype, ISS staging system (Table II). However, no significant correlations were 
Table II. Correlations between ADAMTS9 promoter methylation and clinicopathological indices of MM patients ( $\mathrm{n}=56$ ).

\begin{tabular}{|c|c|c|c|c|}
\hline \multirow[b]{2}{*}{ Clinical parameters } & \multicolumn{2}{|c|}{ ADAMTS9 methylation status } & \multirow[b]{2}{*}{ Total } & \multirow[b]{2}{*}{ P-value } \\
\hline & Methylated (n=37) & Unmethylated $(n=19)$ & & \\
\hline Gender & & & & 0.7144 \\
\hline Male & 22 & 10 & 32 & \\
\hline Female & 15 & 9 & 24 & \\
\hline Age (years) & & & & 0.4612 \\
\hline$<65$ & 16 & 16 & 32 & \\
\hline$\geq 65$ & 21 & 3 & 24 & \\
\hline MM type & & & & 0.9897 \\
\hline IgG & 16 & 7 & 23 & \\
\hline $\operatorname{IgA}$ & 10 & 6 & 16 & \\
\hline$\kappa$ & 7 & 3 & 10 & \\
\hline$\lambda$ & 2 & 2 & 4 & \\
\hline Non-secretory & 2 & 1 & 3 & \\
\hline ISS stage & & & & 0.9247 \\
\hline I & 12 & 7 & 19 & \\
\hline II & 11 & 5 & 16 & \\
\hline III & 14 & 7 & 21 & \\
\hline White blood cell $\left(x 10^{9}\right)$ & $5.42 \pm 2.47$ & $5.96 \pm 1.81$ & & 0.3947 \\
\hline Hemoglobin (g/l) & $96.59 \pm 21.10$ & $91.85 \pm 25.46$ & & 0.4546 \\
\hline Platelets $\left(\mathrm{x} 10^{9}\right)$ & $166.12 \pm 75.75$ & $191.74 \pm 93.50$ & & 0.2739 \\
\hline $\begin{array}{l}\text { Serum calcium } \\
(\mathrm{mmol} / \mathrm{l})\end{array}$ & $2.30 \pm 0.18$ & $2.28 \pm 0.14$ & & 0.6860 \\
\hline $\begin{array}{l}\text { Serum creatinine } \\
(\mu \mathrm{mol} / \mathrm{l})\end{array}$ & $84.70 \pm 10.26$ & $83.85 \pm 11.65$ & & 0.7734 \\
\hline
\end{tabular}

ADAMTS9, a disintegrin-like and metalloprotease with thrombospondin type I motifs 9; MM, multiple myeloma.

A

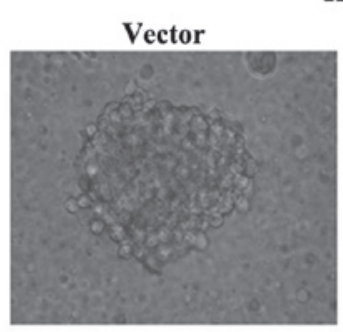

KM3

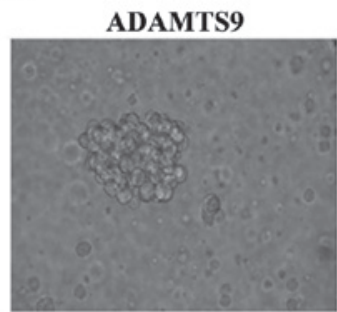

B

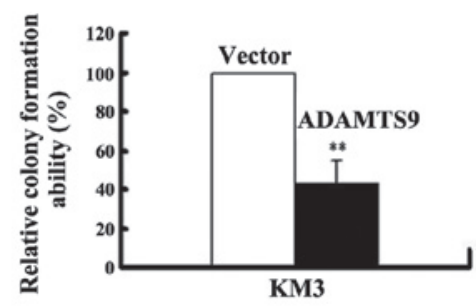

C

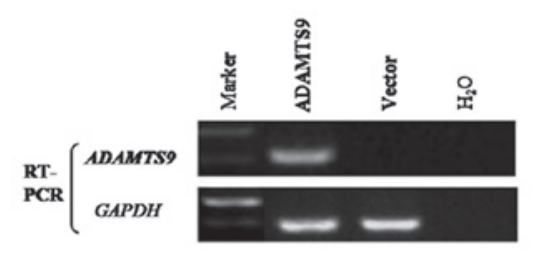

D

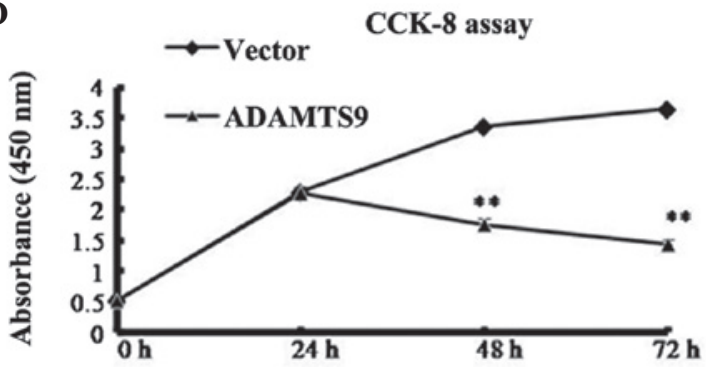

Figure 3. Ectopic expression of ADAMTS9 inhibited clonogenicity of KM3 cells. (A) ADAMTS9 expression inhibited colony formation in soft agar culture. (B) Quantification of colony formation. Number of G418 colonies in the vector-transfected cell line was set to $100 \%$, values are presented as the mean \pm SD. Experiments were performed in triplicate wells 3 times. (C) RT-PCR confirmation of ectopic transcript expression of ADAMTS9 in transfected KM3 cells. (D) CCK-8 cell proliferation assay of vector- and ADAMTS9-transfected KM3 cells. "P $>0.01$, vs. control vector. ADAMTS9, a disintegrin-like and metalloprotease with thrombospondin type I motifs 9. 
A

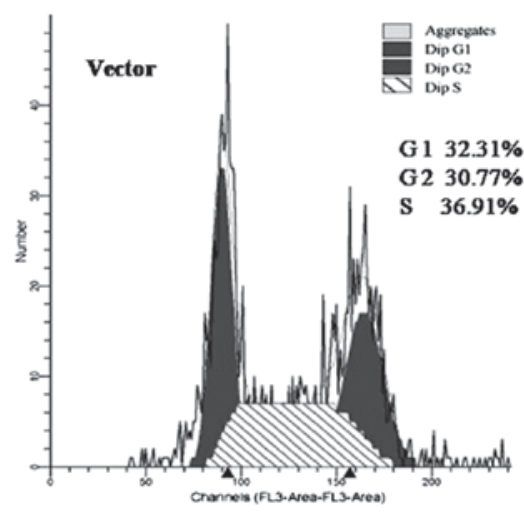

KM3

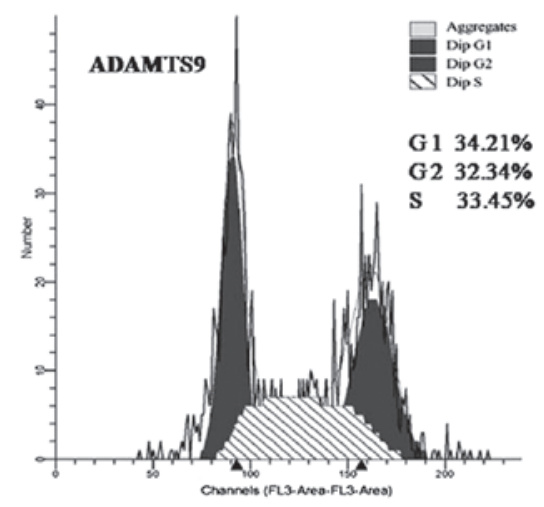

B

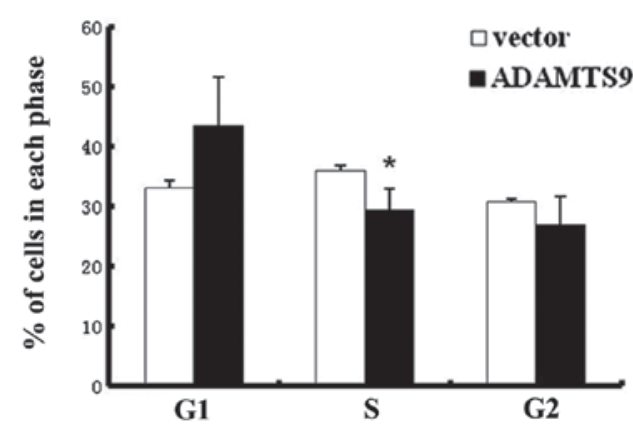

Figure 4. ADAMTS9 induced S-phase cell cycle arrest in KM3 cells. (A) Representative distribution of the cell cycle profile in KM3 cells transfected with or without ADAMTS9 as assessed by fluorescence-activated cell sorting analysis. (B) Analysis of KM3 cells transfected with or without ADAMTS9 distributed in various phases of the cell cycle. The results are presented as the mean \pm SD. $(n=3)$. P $<0.05$, vs. vector control. ADAMTS9, a disintegrin-like and metalloprotease with thrombospondin type I motifs 9.

observed between the patients with methylated ADAMTS9 and these clinicopathological characteristics.

Ectopic expression of ADAMTS9 suppresses tumor cell clonogenicity. The frequent silencing of ADAMTS9 in MM cell lines compared with its broad expression in normal tissues indicates that ADAMTS9 may exert tumor suppressor functions in this tumor type. To verify this hypothesis, a colony formation assay was performed to test for the growth-inhibitory effect of ADAMTS9 in KM3 cells with complete methylation and silencing of ADAMTS9 (Fig. 3A). The ADAMTS9-expressing and empty vector (control) were transfected into KM3 cells. The cells were then selected with $400 \mu \mathrm{g} / \mathrm{ml}$ G418 (Merck, Darmstadt, Germany) to obtain stably transfected cells. Following G418 selection for 2 weeks, ADAMTS9 was stably overexpressed as revealed by RT-PCR (Fig. 3C). A sharp reduction in colonies $(40-60 \%$ of the control vector; $\mathrm{P}<0.01)$ was observed in the KM3-ADAMTS9 cells compared with the empty vector control (Fig. 3B). These results indicate that ADAMTS9 suppresses colony formation and may act as a tumor suppressor in MM cells.

Ectopic expression of ADAMTS9 inhibits tumor cell proliferation and induces $S$-phase cell cycle arrest. The CCK-8 assay was employed to examine the effect of ADAMTS9 on MM cell proliferation in vitro. The growth of ADAMTS9- and vector-expressing KM3 cells was observed at 0, 24, 48 and $72 \mathrm{~h}$. The proliferation trend was identified to be significantly decreased in ADAMTS9-expressing KM3 cells at 48 and $72 \mathrm{~h}$ $(\mathrm{P}<0.01)$ but increased in the vector-expressing KM3 cells in a time-dependent manner (Fig. 3D). To examine the mechanism by which ADAMTS9 inhibits the proliferation of KM3 cells in vitro, the effect of ADAMTS9 expression on cell cycle distribution was investigated using flow cytometry (Fig. 4A). As demonstrated in Fig. 4B, the proportion of cells in the $\mathrm{S}$ phase decreased significantly in ADAMTS9-transfected KM3 cells, which was accompanied by an increase in cells in the $G_{1}$ phase compared with the empty vector-transfected KM3 cells $(\mathrm{P}<0.05)$. These observations are consistent with the hypothesis that the inhibitory effect of cell proliferation induced by ADAMTS9 expression is mediated by cell cycle arrest in the $\mathrm{S}$ phase.

\section{Discussion}

Aberrant promoter methylation may be important in the carcinogenic process (17). Previously, a number of genes, including PTGS2, SFN, CDKN2B, CDH1, ESR1, HIC1, CCND2 and TGF $\beta R 2$, were found to be silenced in association with aberrant methylation in MM (18). Analysis of esophageal and nasopharyngeal cancer demonstrated that the aberrant methylation of ADAMTS9 is important for malignancy (13). However, ADAMTS9 methylation has not been detected in MM. 
The present study confirms that ADAMTS9 is abundantly expressed in normal adult BM samples and RPMI-8226 cells but silenced in KM3 cells and MM BM samples, thereby indicating that ADAMTS9 plays a vital role in the pathogenesis of MM. This study revealed that promoter methylation of ADAMTS9 occurs in KM3 cells and in 66\% of MM samples. These observations indicate that the disruption of ADAMTS9 in MM is markedly associated with aberrant promoter methylation. However, methylation was not detected in RPMI-8226 cells. This demonstrates that methylation of ADAMTS9 is not the only cause of MM and the involvement of additional mechanisms cannot be excluded. Therefore, further studies are required to understand the mechanism of MM development.

Previously, ADAMTS9 expression was found to significantly correlate with lymph node metastases in nasopharyngeal carcinoma (14). However, a significant association was not observed between the methylation status of ADAMTS9 and the clinicopathological indices of our $56 \mathrm{MM}$ patients, indicating that ADAMTS9 methylation may be an early event in MM tumorigenesis. However, the clinical impact of ADAMTS9 inactivation via promoter methylation in MM remains unknown. To assess the correlation between methylation levels of ADAMTS9 with the clinical parameters, studies with larger cohorts, as well as more potential diagnostic and prognostic clinical features are required. ADAMTS9 promoter methylation was detected at a high frequency in MM tissues but not in normal tissues, thereby demonstrating that ADAMTS9 may be a potential cancer-specific biomarker for MM diagnosis. To the best of our knowledge, this is the first study to identify that ADAMTS9 is epigenetically silenced in human MM.

Silencing of ADAMTS9 may be activated by pharmacological demethylation, suggesting that aberrant promoter methylation is a mechanism for ADAMTS9 silencing in MM cells. In the current study, colony-formation, flow cytometry and CCK-8 assay results revealed that as a tumor suppressor, ADAMTS9 suppresses tumor cell clonogenicity and cell proliferation, which may be mediated by cell cycle arrest at the S phase following ADAMTS9 re-expression. Lung et al (14) demonstrated that a marked reduction in the colony-forming ability was observed following transfection of ADAMTS9 into NPC cell lines, which is consistent with our study.

ADAMTS9, together with other members of the ADAMTS family, is involved in tumorigenesis (19-23), which may be associated with anti-angiogenic activity (13, 24-26). Previously, ADAMTS9 was revealed to be a cell-autonomous angiogenesis inhibitor (27). In addition, ADAMTS9 has been identified to function in angiogenesis, however, its role in MM tunorigenesis remains unclear. Further analyses must be conducted to improve understanding of the underlying mechanism.

In conclusion, ADAMTS9 was identified to be frequently silenced by promoter methylation in MM in a cancer-specific manner, which may be reversed by treatment with a demethylation reagent. The transfection of ADAMTS9 into MM cells lacking ADAMTS9 expression led to a reduction in cell colony forming ability and cell proliferation mediated by cell cycle arrest in the $\mathrm{S}$ phase, indicating that epigenetic inactivation of ADAMTS9 is an important factor in MM carcinogenesis. ADAMTS9 may be a candidate tumor suppressor in MM. Further studies are underway to evaluate the possible application of ADAMTS9 as a biomarker for the diagnosis and treatment of MM and to explore its possible impact on the pathways involved in MM development.

\section{Acknowledgements}

The authors thank Dr Jian Hou (The Second Military Medical University, Shanghai, China) for providing the MM cells and the Laboratory of Hematology (the First Affiliated Hospital of Chongqing Medical University) for providing BM samples. The present study was supported by grants from the National Natural Science Foundation of China (nos. 31171243 and 81072148).

\section{References}

1. Hatzimichael E, Dasoula A, Benetatos L, et al: Study of specific genetic and epigenetic variables in multiple myeloma. Leuk Lymphoma 51: 2270-2274, 2010.

2. Rajumar SV and Greipp PR: Prognostic factors in multiple myeloma. Hematol Oncol Clin North Am 13: 1295-1314, 1999.

3. Kyle RA and Rajkumar SV: Multiple myeloma. N Engl J Med 351: 1860-1873, 2004.

4. Klein U, Jauch A, Hielscher T, et al: Chromosomal aberrations $+1 \mathrm{q} 21$ and del(17p13) predict survival in patients with recurrent multiple myeloma treated with lenalidomide and dexamethasone. Cancer 117: 2136-2144, 2011.

5. Esteller M: Epigenetics in cancer. N Engl J Med 358: 1148-1159, 2008.

6. Jost E, Gezer D, Wilop S, et al: Epigenetic dysregulation of secreted Frizzled-related proteins in multiple myeloma. Cancer Lett 281: 24-31, 2009.

7. Boultwood J and Wainscoat JS: Gene silencing by DNA methylation in haematological malignancies. Br J Haematol 138: 3-11, 2007.

8. Yuregir OO, Yurtcu E, Kizilkilic E, Kocer NE, Ozdogu H and Sahin FI: Detecting methylation patterns of p16, MGMT, DAPK and E-cadherin genes in multiple myeloma patients. Int J Lab Hematol 32: 142-149, 2010.

9. Braggio E, Maiolino A, Gouveia ME, et al: Methylation status of nine tumor suppressor genes in multiple myeloma. Int J Hematol 91: 87-96, 2010.

10. Ullmannova-Benson V, Guan M, Zhou X, et al: DLC1 tumor suppressor gene inhibits migration and invasion of multiple myeloma cells through RhoA GTPase pathway. Leukemia 23: 383-390, 2009.

11. Galm O, Yoshikawa H, Esteller M, Osieka R and Herman JG: SOCS-1, a negative regulator of cytokine signaling, is frequently silenced by methylation in multiple myeloma. Blood 101: 2784-2788, 2003.

12. Levy GG, Nichols WC, Lian EC, et al: Mutations in a member of the ADAMTS gene family cause thrombotic thrombocytopenic purpura. Nature 413: 488-494, 2001.

13. Lo PH, Lung H, Cheung AK, et al: Extracellular protease ADAMTS9 suppresses esophageal and nasopharyngeal carcinoma tumor formation by inhibiting angiogenesis. Cancer Res 70: 5567-5576, 2010.

14. Lung HL, Lo PH, Xie D, et al: Characterization of a novel epigenetically-silenced, growth-suppressive gene, ADAMTS9 and its association with lymph node metastases in nasopharyngeal carcinoma. Int J Cancer 123: 401-408, 2008.

15. Zhang C, Shao Y, Zhang W, et al: High-resolution melting analysis of ADAMTS9 methylation levels in gastric, colorectal and pancreatic cancers. Cancer Gene Cytogenet 196: 38-44, 2010.

16. Kuo HK, Griffith JD and Kreuzer KN: 5-Azacytidine induced methyltransferase-DNA adducts block DNA replication in vivo. Cancer Res 17: 8248-8254, 2007.

17. Sidransky D: Emerging molecular markers of cancer. Nat Rev Cancer 2: 210-219, 2002. 
18. de Carvalho F, Colleoni GW, Almeida MS, Carvalho AL and Vettore AL: TGF $\beta$ R2 aberrant methylation is a potential prognostic marker and therapeutic target in multiple myeloma. Int J Cancer 125: 1985-1991, 2009.

19. Lo PH, Leung AC, Kwok CY, et al: Identification of a tumor suppressive critical region mapping to 3 p14.2 in esophageal squamous cell carcinoma and studies of a candidate tumor suppressor gene, ADAMTS9. Oncogene 26: 148-157, 2007.

20. Li Z, Zhang W, Shao Y, et al: High-resolution melting analysis of ADAMTS18 methylation levels in gastric, colorectal and pancreatic cancers. Med Oncol 27: 998-1004, 2010.

21. Moncada-Pazos A, Obaya AJ, Fraga MF, et al: The ADAMTS12 metalloprotease gene is epigenetically silenced in tumor cells and transcriptionally activated in the stroma during progression of colon cancer. J Cell Sci 122: 2906-2913, 2009.

22. Viloria CG, Obaya AJ, Moncada-Pazos A, et al: Genetic inactivation of ADAMTS15 metalloprotease in human colorectal cancer. Cancer Res 69: 4926-4934, 2009.
23. Dunn JR, Panutsopulo D, Shaw MW, et al: METH-2 silencing and promoter hypermethylation in NSCLC. Br J Cancer 91: 1149-1154, 2004

24. Dubail J, Kesteloot F, Deroanne C, et al: ADAMTS-2 functions as anti-angiogenic and anti-tumoral molecule independently of its catalytic activity. Cell Mol Life Sci 67: 4213-4232, 2010.

25. EI Hour M, Moncada-Pazos A, Blacher S, et al: Higher sensitivity of Adamts12-deficient mice to tumor growth and angiogenesis. Oncogene 29: 3025-3032, 2010.

26. Dunn JR, Reed JE, du Plessis DG, et al: Expression of ADAMTS-8, a secreted protease with antiangiogenic properties, is downregulated in brain tumours. Br J Cancer 94: 1186-1193, 2006.

27. Koo BH, Coe DM, Dixon LJ, et al: ADAMTS9 is a cell-autonomously acting, anti-angiogenic metalloprotease expressed by microvascular endothelial cells. Am J Pathol 176: 1494-1504, 2010. 